\title{
Niveles de ansiedad durante el confinamiento obligatorio por covid-19, en estudiantes de una Universidad Estatal del Caribe colombiano
}

\author{
Anxiety levels during compulsory confinement due to \\ covid-19, in students of a Colombian Caribbean State \\ University
}
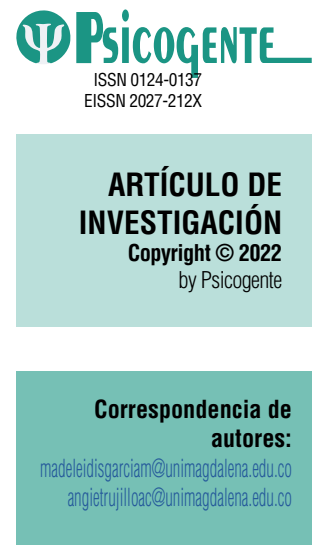

Recibido: 26-08-20 Aceptado: 24-01-22 Publicado: $01-04-22$

\section{Níveis de ansiedade durante o confinamento obrigatório devido ao covid-19, em alunos de uma Universidade Estadual do Caribe colombiano.}

Madeleidis García Muñoz (iD - Angie Trujillo Camacho (iD

Universidad del Magdalena, Santa Marta, Colombia

\section{RESUMEN}

Introducción: La alarma mundial por la pandemia del covid-19 ha afectado no solo la salud física sino también la salud mental de toda la población, incluyendo a los jóvenes universitarios.

Objetivo: Caracterizar la presencia de la ansiedad (Ansiedad Estado-AE y Ansiedad Rasgo-AR) en estudiantes de una Universidad estatal del Caribe colombiano en situación de confinamiento obligatorio por el covid-19.

Método: investigación cuantitativa de tipo descriptiva y diseño transversal, con muestreo intencional Participaron 371 estudiantes de las seis Facultades de la universidad participante, hombres y mujeres, con edad mínima de 16 y máxima de 33 años, M de 18,3 años y DT de 2,1 años. Los estudiantes respondieron el Cuestionario STAI (State Trait Anxiety Inventory), mediante la plataforma de Google Forms, el cual fue enviado a 1200 estudiantes por medio de correo electrónico, durante el mes de mayo de 2020. Los datos fueron procesados mediante el SPSSv21, se hicieron análisis descriptivos por grupos y de Odds Ratio (OR).

Resultados: Se hallaron niveles de ansiedad, ligeramente superior en AE y AR en el sexo masculino en la categoría muy alto tanto para adolescentes como adultos. Mientras que, en la categoría alta, se presentó mayor AE en el sexo femenino en el rango de edad de 19 años o más, y en el rango de 16 a 18 años AR ligeramente más alta en el sexo femenino. Así mismo, las mujeres adolescentes tienen un OR de 2,2 veces más de $A E$ que las adultas, representando un riesgo para su salud.

Conclusiones: Los estudiantes universitarios presentan altos niveles de ansiedad, ligeramente mayor en los adolescentes respecto a los adultos, lo que pone en riesgo su salud mental, por lo que se sugiere medidas de intervención desde la psicología de la salud con fines de prevención y monitorear los avances mediante nuevas investigaciones.

Palabras clave: ansiedad, confinamiento obligatorio, aislamiento social, covid-19, estudiantes universitarios.

\section{ABSTRACT}

Introduction: The global alarm for the covid-19 pandemic has affected not only the physical health but also the mental health of the entire population, including young university students.

Objective: To characterize the presence of anxiety (Anxiety State-AE and Anxiety trait-AR) in students of a Colombian Caribbean State University in a situation of compulsory confinement due to covid-19.

Method: descriptive quantitative research and cross-sectional design, with intentional sampling. 371 students from the six Faculties of the participating university participated, men and women, with 
a minimum age of 16 and a maximum of 33 years, $M$ of 18,3 years and DT of 2,1 years. The students responded to the STAI Questionnaire (State Trait Anxiety Inventory), through the Google Forms platform, which was sent to 1200 students by email, during the month of May 2020. The data was processed through the SPSSv21, they did descriptive analysis by groups and Odds Ratio (OR).

Results: Anxiety levels were found to be slightly higher in EA and RA in the male sex in the very high category for both adolescents and adults. Meanwhile, in the high category, higher EA was found in the female sex in the age range of 19 years or older, and in the range of 16 to 18 years RA was slightly higher in the female sex. Likewise, adolescent females have an OR of 2,2 times more EA than adults, representing a risk to their health.

Conclusions: University students present high levels of anxiety, slightly higher in adolescents than in adults, which puts their mental health at risk, therefore, intervention measures are suggested from health psychology for prevention and monitoring purposes advances through new research.

Keywords: anxiety, compulsory confinement, social isolation, covid-19, university students.

\section{RESUMO}

Introdução: O alarme global sobre a pandemia covid-19 afetou não apenas a saúde física, mas também a saúde mental de toda a população, incluindo jovens estudantes universitários.

Objetivo: Caracterizar a presença de ansiedade (Ansiedade do Estado-AE e Ansiedade Traço-AR) em alunos de uma Universidade Estadual do Caribe colombiano em situação de confinamento obrigatório por covid-19.

Método: pesquisa quantitativa do tipo descritivo e delineamento transversal, com amostragem intencional. Participaram 371 alunos das seis Faculdades da universidade participante, homens e mulheres, com idade mínima de 16 e máxima de 33 anos, M de 18,3 anos e DT de 2,1 anos. Os alunos responderam ao STAI Questionnaire (State Trait Anxiety Inventory), por meio da plataforma Google Forms, que foi enviado a 1200 alunos por e-mail, durante o mês de maio de 2020. Os dados foram processados por meio do SPSSv21, fez análise descritiva por grupos e Odds Ratio (OR).

Resultados: Foram encontrados níveis ligeiramente mais elevados de ansiedade na AE e na AR em homens da categoria muito alta, tanto para adolescentes como para adultos. Na categoria alta, a AE era mais elevada nas fêmeas na faixa etária dos 19+, e na faixa etária dos 16-18, a RA era ligeiramente mais elevada nas fêmeas. Do mesmo modo, as fêmeas adolescentes têm um OR 2,2 vezes mais AE que os adultos, representando um risco para a sua saúde.

Conclusões: Os universitários apresentam níveis elevados de ansiedade, ligeiramente superiores nos adolescentes do que nos adultos, o que põe em risco a sua saúde mental, pelo que são sugeridas medidas de intervenção da psicologia da saúde para efeitos de prevenção e acompanhamento avanços por meio de novas pesquisas.

Palavras-chave: ansiedade, confinamento obrigatório, isolamento social, covid-19, estudantes universitários.

\section{INTRODUCCIÓN}

La enfermedad por Coronavirus o covid-19 se originó a principios de diciembre de 2019 en Wuhan, China, y se propagó rápidamente en enero de 2020 llegando a más de 118 países; para tal efecto, el número de contagiados y muertes fue alarmante por lo que este nuevo virus fue considerado como emergencia de salud pública a nivel mundial (Yin, Feng, Wang, Chen, Wu, Chen, Lv y Xiang, 2020). El covid-19 es altamente infeccioso y causa una enfermedad respiratoria como la gripe (influenza), con diferentes síntomas como fiebre, escalofríos, tos, dolor de garganta, dificultad para respirar, etc., y en casos graves producir una neumonía que puede llevar a la muerte (Wang, Pan, Wan, Tan, Xu, Ho y Ho, 2020). 
Conviene señalar que, existía poca información alrededor de este nuevo virus, por lo que no había una vacuna para prevenirlo, ni un método de tratamiento específico que fuera efectivo, en vista de lo anterior, para controlar su propagación, el gobierno chino recomendó adoptar una serie de medidas estrictas de prevención y control, como es el cierre de ciudades y el aislamiento obligatorio que restringía a las personas de entrar y salir de sus hogares (Yin, Wang, Feng et al., 2020).

De ahí que, ante la llegada del primer caso por covid-19 a América Latina, se activaron los protocolos y alarmas sanitarias, aun así, los casos confirmados y de fallecidos fueron aumentando y se extendió en diferentes países, constatándose el primer caso en Colombia el 6 de marzo de 2020, por esta razón, el Ministerio de Salud y Protección Social declaró emergencia sanitaria por causa del coronavirus en todo el territorio nacional y se establecieron diferentes medidas sanitarias con el objetivo de prevenir y controlar la propagación.

Una de las medidas preventivas fue el aislamiento obligatorio para todos los colombianos, que iría desde el 24 de marzo hasta el 13 de abril de 2020, no obstante, este periodo se fue ampliando cada vez más, debido al aumento de contagiados en el país. Todos estos hechos ciertamente tienen un gran impacto psicológico, ya que obligan a que las personas adopten nuevas formas de comportamientos y hábitos diarios ante el peligro inminente de contagio.

En efecto, el impacto del covid-19 en la población general confinada es alarmante, tal como se encontró en una encuesta realizada a 1.210 personas en China, donde se reportó que el 53,8 \% valoró el impacto psicológico de la situación como moderado-grave, un 16,5 \% refirió síntomas depresivos entre moderados y graves, un $28,8 \%$ síntomas de ansiedad entre moderados y graves, y un $8,1 \%$ niveles de estrés entre moderados y graves. La mayoría de los participantes encuestados $(84,7 \%)$ permanecieron confinados en casa entre 20 y 24 horas al día y su principal preocupación (75,2 \%) fue que sus familiares se contagiaran de covid-19 (Wang, Pan, Wan, Tan, Xu, Ho y Ho, 2020).

El rápido crecimiento de casos confirmados de contagio y muertes por covid-19 a pesar del confinamiento obligatorio, ha evidenciado la presencia de diversos problemas psicológicos, como la ansiedad, depresión y estrés, sobre todo en la fase inicial de la pandemia, debido a que la información científica que se lleva tanto a la población general como al personal de salud, se centra en los aspectos genéticos y epidemiológicos, dejando de lado la salud mental, por lo que propone la necesidad de atender esta a fin de prevenir su impacto en la salud y lograr controlar los efectos que trae consigo la pandemia (Huarcaya, 2020). 
Ahora bien, en Colombia, desde el mes de marzo de 2020 se ordenó el confinamiento obligatorio, con la consecuente decisión del Ministerio de Educación de autorizar la modificación de la educación superior hacia la modalidad remota o asistida por tecnología de la Información, con el fin de garantizar el derecho a la educación de sus ciudadanos. Sin embargo, esta decisión ha generado un fuerte impacto en el estilo de vida y la salud mental de estudiantes y docentes, cuyas consecuencias apenas se está estudiando.

Debido a este confinamiento obligatorio, el sistema educativo se ha visto afectado, y por lo tanto, impuesto la necesidad de modificar de manera brusca, intempestiva, nada planificada, la educación virtual o también llamada clases remotas asistidas por tecnologías de la información y la comunicación, Tic.

En consecuencia, los estudiantes universitarios al estar expuestos a situaciones de estrés y de incertidumbre tanto en el ámbito académico, como en el social y familiar, se convierten en una población en riesgo de presentar altos niveles de ansiedad, debido a que, sus prácticas diarias, incluidas las académicas, han cambiado. Por consiguiente, algunos estudiantes podrían presentar ansiedad frente a los compromisos académicos que no cesan, al constantemente interés por recibir información acerca del covid-19, la falta de recursos económicos, falta de actividad física y la monotonía (GonzálezJaimes, Tejeda-Alcántara, Espinosa-Méndez, Ontiveros-Hernández, 2020).

Las funciones misionales de la Universidad, implican el desarrollo de una serie de actividades académicas complejas y permanentes de estudiantes y docentes, que pueden desencadenar niveles de estrés y ansiedad, afectando la salud mental de estas poblaciones. Esta situación de estrés académico ha sido reconocida en diferentes estudios desde antes de la aparición de la pandemia mundial por covid-19, que conllevó a que los países entrasen en confinamiento obligatorio (Talavera, 2020; Cobo-Rendón, Vega-Valenzuela y García Álvarez, 2020; Cáceres, 2020).

En cuanto a la ansiedad, esta es definida como una respuesta anticipatoria a un peligro real o imaginario que lleva a la persona a presentar conductas de evitación o de escape y que en ocasiones puede manifestarse en una respuesta excesiva, debido a la sensación de no poder controlar dicho peligro, llevándolo finalmente a un mayor miedo, ansiedad y otras conductas similares (Raines, Oglesby, Unruh, Capron y Schmidt, 2013; citados por Quezada-Scholz, 2020), lo que podría a su vez generar una respuesta maladaptativa, nada saludable o éticamente cuestionable. 
La ansiedad, puede presentarse de dos maneras o de acuerdo con dos factores: como estado o como rasgo. La AE se define como un estado o condición emocional transitoria de la persona, que se caracteriza por sentimientos subjetivos, conscientemente percibidos, de tensión y aprehensión, así como por una hiperactividad del sistema nervioso autonómico; la cual puede variar con el tiempo y fluctuar en intensidad. De otra parte, la Ansiedad Rasgo (AR) se concibe como una propensión ansiosa relativamente estable, por lo que las personas difieren en su tendencia a percibir las situaciones como amenazadoras y a elevar, consecuentemente, su Ansiedad Estado (AE). (Spielberger, Gorsuch y Lushene, 1997).

Es indudable que el factor psicológico es relevante y debe ser considerado en la comprensión, control y superación de la pandemia por el covid-19, pues la experiencia ha demostrado que aunque el factor biológico es esencial (virus, mecanismos biológicos involucrados y tratamiento farmacológico u otros disponibles), los factores psicológicos y sociales son determinantes, puesto que afecta diversos aspectos de la vida de las personas, las familias, las comunidades y la sociedad en general modificando su vida cotidiana, (Vera-Villarroel y Vogel, 2010; Vera-Villarroel, Celis-Atenas, Córdova-Rubio, Zych y Buela-Casal, 2011; citados en Urzúa, Vera-Villarroel, Caqueo-Urízar y Polanco-Carrasco, 2020).

Por cierto, las respuestas más comunes y generalizadas que se han experimentado durante el brote y riesgo del coronavirus han sido el miedo y la incertidumbre; emociones que al mismo tiempo son un probable factor de riesgo y contagio propio y de otros. Estas respuestas difieren entre las personas y pueden expresarse desde una respuesta de miedo y ansiedad óptima acorde con la situación de amenaza, hasta estados emocionales desproporcionados y prolongados (incluso irreversibles), experimentados como miedo y ansiedad generalizados excesivos, persistentes y/u omnipresentes (Wojdat y Pape, 2013). Pero, cuando el miedo se generaliza a situaciones que no son una amenaza real llega a ser patológico, donde el individuo sufre una experiencia desagradable dado que tiende a evaluar un estímulo o evento como peligroso, aunque realmente no lo sea, por lo que busca evitar una amplia variedad de estímulos y situaciones, constituyéndose este hecho en un verdadero trastorno ansioso, uno de los más prevalentes y debilitantes de los trastornos psiquiátricos (Olatunji, Cisler, y Tolin, 2007; citados en Quezada-Scholz, 2020).

Cabe aclarar que, según Lazarus (1991), no es el estresor el causante del estrés sino la percepción que tiene la persona sobre dicho estresor; $y$, asimismo, 
las expectativas del individuo sobre sus metas o exigencias ambientales, también pueden ser percibidas en forma positiva como desafíos o negativa como amenaza y, por tanto, reaccionar en consecuencia.

El riesgo de sufrir altos niveles de estrés y ansiedad en la actividad académica universitaria ha sido reconocido en diversos estudios desde antes del inicio de la pandemia, tanto en ambientes presenciales (Barraza y Jaick, 2011; Ferrel, Ferrel, Cantillo, Jaramillo y Jiménez, 2017), como en situaciones mediadas por las Tics, tal como se reporta en el estudio realizado por Ortiz, Tenezaca, Torres y Ugalde, (2014), sobre la relación entre el uso excesivo de las Tics y la presencia de síntomas de depresión y ansiedad en estudiantes de 2 año de Enfermería y Tecnología Médica de la Facultad de Medicina; se halló que las situaciones generadoras de estrés fueron similares para ambos grupos, pero varias de ellas fueron percibidas como estresores significativamente mayores por los estudiantes de Enfermería, siendo tres las principales fuentes de estrés: la sobrecarga académica, falta de tiempo para cumplir con las actividades académicas y realización de un examen.

De otra parte, en un estudio realizado para determinar los niveles de ansiedad presentes en estudiantes de medicina en Chile, encontraron que los estudiantes de enfermería presentaron mayores niveles de $A E$ y $A R$ que los estudiantes de Tecnología Médica y, aunque las situaciones generadoras de estrés fueron similares para ambos grupos, varias fueron percibidas como estresores significativamente mayores por las estudiantes de enfermería, por lo que sugieren desarrollar consejería y talleres para el adecuado afrontamiento del estrés académico (Castillo, Chacón de la Cruz y Díaz-Véliz, 2016).

En un estudio realizado en la Comunidad Autónoma Vasca al norte de España, se analizaron los niveles de estrés, ansiedad y depresión en el periodo de llegada del virus, junto con los niveles de sintomatología psicológica, según edad, cronicidad y confinamiento, en una muestra de 976 personas, hallándose que, aunque los niveles de sintomatología fueron bajos, la población más joven y con enfermedades crónicas reportaron sintomatología más alta que el resto de la población. También encontraron un mayor nivel de sintomatología a partir del confinamiento y esperan que la sintomatología aumente según vaya transcurriendo más tiempo, por lo que proponen intervenciones psicológicas de prevención y tratamiento para reducir este impacto psicológico (Ozamiz-Etxebarria, Dosil-Santamaria, Picaza-Gorrochategui y IdoiagaMondragón, 2020). 
Similares resultados se hallaron en un estudio realizado en Puerto Rico, con el objetivo de evaluar las demandas tecnológicas, académicas, y psicológicas experimentadas por una muestra de 167 estudiantes universitarios de 17 instituciones de educación superior, durante el periodo de la pandemia por covid-19. Los hallazgos más destacados que se reportan señalan la presencia de problemáticas relacionadas con estresores debido a la sobrecarga de tareas, mala implementación de cursos en línea, falta de orientación de sus universidades, poca flexibilidad de sus profesores, entre otras, por lo que sugieren continuar con estudios sobre el repentino cambio de modalidades educativa, de presencial a virtual o remoto y sus efectos en los estudiantes (Rivera, Cruz-Santos y Rodríguez-Ríos, 2020).

De acuerdo con lo anterior, se puede afirmar que la incertidumbre que se tiene sobre la enfermedad y las consecuencias que esta trae consigo, tienen unos efectos psicológicos entre las personas, ya que a medida que la enfermedad se propaga y aumentan las casos confirmados y muertes en el mundo, así mismo aumenta el miedo y la ansiedad en ellos. Así pues, los niveles de ansiedad pueden variar dependiendo de las actividades diarias que realicen las personas mientras se encuentran en aislamiento social y a la cantidad de información a la que se encuentran expuestos diariamente.

En consideración a lo expuesto, los investigadores se plantearon el objetivo de caracterizar los niveles de ansiedad presentes en los estudiantes universitarios de una Universidad estatal del Caribe colombiano, en periodo de confinamiento obligatorio debido a la pandemia del covid-19, dado que aún no hay datos concluyentes sobre la presencia de esta variable en contexto de la pandemia en la población universitaria en Colombia, a partir de las cuales se puedan esbozar algunas estrategias de intervención, encaminadas a disminuir la sintomatología ansiosa que presenten los estudiantes durante la crisis.

\section{MÉTODO}

\subsection{Diseño}

Con el fin de cumplir con los objetivos planteados, se aplicó una investigación cuantitativa de tipo descriptiva y de diseño transversal, mediante el cual se aplicó el instrumento de investigación vía on line durante el mes de mayo de 2020. 


\subsection{Participantes}

Se tomó como población a los estudiantes de la Universidad del Magdalena, ubicada en la ciudad de Santa Marta, Colombia; tomando como muestra a 1.200 estudiantes, escogidos mediante muestreo por conveniencia, los cuales fueron seleccionados con base al acceso a sus correos electrónicos institucionales. Al cuestionario respondieron 374 estudiantes, pero al hacer la depuración de la matriz se eliminaron 3 sujetos, ya que no tenían los datos completos, quedando 371 participantes. Colaboraron estudiantes de las seis Facultades de la Universidad: Ciencias Básicas (6\%), Ciencias Económicas y Empresariales (32\%), Ciencias de la Salud (13\%), Ingeniería (32\%), Humanidades (16 \%) y Educación (1\%). De ellos, 153 hombres (41\%) y 218 mujeres (59\%); en cuanto a edades, se dividieron en dos grupos, 245 (66\%) tenían de 16 a 18 años y 126 (34 \%) tenían de 19 años a más; con edad mínima de 16 y máxima de 33 años, Media de 18,3 años y Desviación Típica de 2,1 años. Se hallaron diferencias respecto a la proporción de participantes según sexo en algunas Facultades como Ingeniería, donde hubo mayor representación del sexo femenino (80 \%) que del masculino (20\%); y, escasa participación (3 sujetos) en la Facultad de Ciencias de la Educación, lo que hizo difícil la realización de análisis significativos.

\subsection{Instrumento}

El instrumento aplicado (State Trait Anxiety Inventory, STAI, Spielberger, Gorsuch y Lushene, 1970) mide dos dimensiones: Ansiedad Estado (AE) y Ansiedad rasgo (AR).

De acuerdo con sus autores, la $\mathrm{AE}$ se define como un estado o condición emocional transitoria de la persona, que se caracteriza por sentimientos subjetivos, conscientemente percibidos, de tensión y aprehensión, así como por una hiperactividad del sistema nervioso autonómico; la cual puede variar con el tiempo y fluctuar en intensidad. De otra parte, la AR se concibe como una propensión ansiosa relativamente estable, por lo que las personas difieren en su tendencia a percibir las situaciones como amenazadoras y a elevar, consecuentemente, su ansiedad Estado (AE).

EI STAI consta de 40 ítems, cada una de las dos subescalas cuenta con 20 ítems. Los ítems se formulan haciendo referencia tanto a la ausencia como a la presencia de ansiedad, en ambas subescalas y se responden mediante una escala Likert, de 0 a 3, en cuatro categorías: muy alta, alta, media y baja. Las puntuaciones finales pueden ir de 20 a 80 , donde a mayor puntaje mayor ansiedad manifestará la persona. En la adaptación española, el STAI tiene una 
alta consistencia interna por subescala, con un alfa de Cronbach entre 0,9 y 0,93 para AE y entre 0,84 y 0,87 para AR (Spielberger et al., 1997, p.22).

\subsection{Análisis de Resultados}

Para el análisis de los resultados se dividió a los participantes en dos grupos según rangos de edad, de 16 a 18 años (adolescentes, de acuerdo con los baremos del STAI), y de 19 años o más (adultos, baremos STAI); y, para la interpretación de los resultados se siguieron los baremos señalados en el Manual de la Prueba STAI, en cuatro categorías: muy alta, alta, media y baja. Los datos fueron procesados mediante el SPSSv21, se hicieron análisis descriptivos por grupos y de riesgo mediante el estadístico Odds Ratio (OR).

\subsection{Procedimiento}

El instrumento aplicado State Trait Anxiety Inventory (STAI), fue adaptado a la plataforma de Google Forms y se difundió vía on line, enviando 1.200 correos electrónicos a los correos institucionales de los estudiantes participantes, ya que estos se encontraban en sus casas por confinamiento obligatorio debido a la pandemia del covid-19 (https://www.google.com/intl/es-419/forms/ about/).

\subsection{Aspectos éticos}

Se respetaron las consideraciones éticas consignadas en la Resolución №08430 del 4 de octubre de 1993 del Ministerio de Protección Social de Colombia y en la Ley 1090 del 6 de septiembre de 2006 o Ley del Psicólogo en Colombia, orientados a garantizar la seguridad y bienestar de los participantes de la investigación, total confidencialidad de los datos, información y resultado de las pruebas de medición, entre otras, procedentes del sujeto de investigación. En ese marco legal, los participantes fueron informados sobre el objetivo del estudio, no recibieron dinero, ni puntos-créditos académicos por su participación, y firmaron el Consentimiento Informado formulado al inicio del Cuestionario.

\section{RESULTADOS}

En la realización del estudio participaron 374 estudiantes universitarios, pero al hacer la depuración de la matriz se eliminaron 3 sujetos por carecer de datos completos. Todos respondieron el cuestionario de manera individual, vía on line, estando en sus propias viviendas, debido al confinamiento obligatorio por el covid-19 en que se encontraban. 
Tabla 1.

Estadísticos Variable Edad

\begin{tabular}{|c|c|c|c|c|}
\hline N VÁLIDOS & MEDIA & DESV. TíP. & MÍNIMO & MÁXIMO \\
\hline 371 & 18,31 & 2,167 & 16 & 33 \\
\hline
\end{tabular}

Los participantes tenían una media de edad de 18,3 años, con desviación estándar de 2,22, siendo la edad mínima de 16 años y la máxima de 33 (Tabla 1).

Tabla 2.

Contingencia Edad Actual * Sex

\begin{tabular}{|c|c|c|c|c|}
\hline \multirow{2}{*}{\multicolumn{2}{|c|}{ EDAD ACTUAL }} & \multicolumn{2}{|c|}{ SEXO } & \multirow{3}{*}{$\begin{array}{r}\text { TOTA } \\
245\end{array}$} \\
\hline & & \multirow{2}{*}{$\begin{array}{c}\text { MASCULINO } \\
91\end{array}$} & \multirow{2}{*}{$\begin{array}{c}\text { FEMENINO } \\
154\end{array}$} & \\
\hline \multirow{3}{*}{ De 16 a 18 años } & Recuento & & & \\
\hline & $\%$ dentro de eAct & $37 \%$ & $63 \%$ & $100 \%$ \\
\hline & $\%$ del total & $25 \%$ & $42 \%$ & $66 \%$ \\
\hline \multirow{3}{*}{ De 19 años o más } & Recuento & 62 & 64 & 126 \\
\hline & $\%$ dentro de eAct & $49 \%$ & $51 \%$ & $100 \%$ \\
\hline & $\%$ del total & $17 \%$ & $17 \%$ & $34 \%$ \\
\hline \multirow{3}{*}{ Total } & Recuento & 153 & 218 & 371 \\
\hline & $\%$ dentro de eAct & $41 \%$ & $59 \%$ & $100 \%$ \\
\hline & \% del total & $41 \%$ & $59 \%$ & $100 \%$ \\
\hline
\end{tabular}

Para efectos del análisis, se dividió a los participantes en dos grupos según rangos de edad, de 16 a 18 años (adolescentes, de acuerdo con los baremos del STAI), y de 19 años o más (adultos, baremos STAI). En el primer grupo participaron 245 estudiantes, representando el $66 \%$ de la muestra de estudio; $25 \%$ perteneciente al sexo masculino y $42 \%$ al sexo femenino. En el segundo grupo participaron 126 estudiantes, representando el $34 \%$ de la muestra, teniendo una proporción equitativa del $17 \%$ para los dos sexos (Tabla 2).

Tabla 3.

Contingencia Sexo *Programa

\begin{tabular}{|c|c|c|c|c|c|c|c|c|}
\hline & \multicolumn{8}{|c|}{ FACULTAD } \\
\hline & SEXO & $\begin{array}{l}\text { C. BÁSICAS } \\
\text { BIOLOGÍA }\end{array}$ & $\begin{array}{c}\text { CIENCIAS } \\
\text { EMPRESA ECONOMÍA }\end{array}$ & $\begin{array}{l}\text { CIENCIAS } \\
\text { SALUD }\end{array}$ & INGENIERÍA & HUMANIDADES & $\begin{array}{l}\text { CIENCIAS } \\
\text { EDUCACIÓN }\end{array}$ & TOTAL \\
\hline \multirow{3}{*}{ MASC } & Recuento & 12 & 56 & 25 & 24 & 34 & 2 & 153 \\
\hline & $\begin{array}{l}\text { \% dentro de } \\
\text { facultad }\end{array}$ & $55 \%$ & $47 \%$ & $51 \%$ & $20 \%$ & $59 \%$ & $67 \%$ & $41 \%$ \\
\hline & $\%$ del total & $3 \%$ & $15 \%$ & $7 \%$ & $6 \%$ & $9 \%$ & $1 \%$ & $41 \%$ \\
\hline \multirow{3}{*}{ Fem } & Recuento & 10 & 64 & 24 & 95 & 24 & 1 & 218 \\
\hline & $\begin{array}{l}\text { \% dentro de } \\
\text { facultad }\end{array}$ & $45 \%$ & $53 \%$ & $49 \%$ & $80 \%$ & $41 \%$ & $33 \%$ & $59 \%$ \\
\hline & $\%$ del total & $3 \%$ & $17 \%$ & $6 \%$ & $26 \%$ & $6 \%$ & $0 \%$ & $59 \%$ \\
\hline
\end{tabular}




\begin{tabular}{|c|c|c|c|c|c|c|c|c|}
\hline \multirow{3}{*}{ Total } & Recuento & 22 & 120 & 49 & 119 & 58 & 3 & 371 \\
\hline & $\begin{array}{c}\% \text { dentro de } \\
\text { facultad }\end{array}$ & $100 \%$ & $100 \%$ & $100 \%$ & $100 \%$ & $100 \%$ & $100 \%$ & $100 \%$ \\
\hline & $\%$ del total & $6 \%$ & $32 \%$ & $13 \%$ & $32 \%$ & $16 \%$ & $1 \%$ & $100 \%$ \\
\hline
\end{tabular}

La Tabla 3 reporta que los estudiantes de la Facultad de Ciencias Empresariales y Económicas junto a Ingeniería agruparon el $64 \%$ de la muestra, con 120 sujetos, y 119 sujetos respectivamente ( $32 \%$ cada uno). Seguidos de lejos en cuanto a representación de la Facultad de Humanidades con 58 sujetos (16\% de la muestra), Ciencias de la Salud con 49 sujetos (13\% de la muestra) y Ciencias Básicas (Biología) con 22 sujetos ( $6 \%$ de la muestra). Ciencias de la Educación tuvo la más baja representación con 3 sujetos, representando el $1 \%$ de la muestra de estudio.

Tabla 4.

Baremos para adolescentes para el STAI

\begin{tabular}{ccccc}
\hline ADOLESCENTES & \multicolumn{2}{c}{ VARONES } & \multicolumn{2}{c}{ MUJERES } \\
\hline CATEGORÍAS & AE & AR & AE & AR \\
\hline Muy alta & 32 o más & 27 o más & 32 o más & 30 o más \\
Alta & 25 a 31 & 23 a 26 & 26 a 31 & 26 a 29 \\
Media & 18 a 24 & 1922 & 20 a 25 & 21 a 25 \\
Baja & 0 a 17 & 0 a 18 & 0 a 19 & 0 a 20 \\
\hline
\end{tabular}

Tabla 5.

Baremos para adultos para el STAI

\begin{tabular}{ccccc}
\hline ADULTOS & \multicolumn{2}{c}{ VARONES } & \multicolumn{2}{c}{ MUJERES } \\
\hline CATEGORÍAS & AE & AR & AE & AR \\
\hline Muy alta & 29 o más & 26 o más & 32 o más & 33 o más \\
Alta & 22 a 28 & 23 a 25 & 25 a 31 & 28 a 32 \\
Media & 19 a 21 & 20 a 22 & 21 a 24 & 24 a 27 \\
Baja & De 0 a 18 & 0 a 19 & 0 a 20 & 0 a 23
\end{tabular}

Para la interpretación de los resultados se siguieron los baremos señalados en el Manual de la Prueba STAI, en cuatro categorías: muy alta, alta, media y baja (Tablas 4 y 5), teniendo en cuenta que, en los estudios originales, se encontraron diferencias significativas en la ansiedad en relación con la edad y el sexo, por lo cual establecieron diferentes rangos de puntuaciones teniendo en cuenta estas variables tanto para Ansiedad Estado - $A E$, como para Ansiedad Rasgo - AR (Spielberger, Gorsuch y Lushene, 1997). Así, los datos se ajustaron al contexto colombiano en donde se considera la mayoría de edad legal a los 18 años, pero se incluyó esta edad en el rango de adolescentes, y a partir de los 19 años se consideró que debía iniciar el rango de edad para los sujetos adultos. 


\subsection{Niveles de ansiedad por categorías}

Tabla 6.

Ansiedad de adolescentes 16 a 18 años, Sex = Masculino; 91 sujetos

\begin{tabular}{ccccc}
\hline ADOLESCENTES & \multicolumn{2}{c}{ ANSIEDAD ESTADO } & \multicolumn{2}{c}{ ANSIEDAD RASGO } \\
\hline CATEGORÍAS & FREC & $\%$ & FREC & $\%$ \\
\hline Muy alta & 34 & 37 & 67 & 74 \\
Alta & 41 & 45 & 15 & 16 \\
Media & 13 & 14 & 8 & 9 \\
Baja & 3 & 3 & 1 & 1 \\
Total & 91 & 100 & 91 & 100 \\
\hline
\end{tabular}

De acuerdo con la puntuación obtenida a partir de los baremos establecidos, se hallaron altos niveles de ansiedad en los participantes varones de 16 a 18 años (91 sujetos). El $82 \%$ presentaron AE muy alta y alta (37\% y $45 \%$, respectivamente), mientras que en Ansiedad Rasgo el $\mathbf{9 0} \%$ de los sujetos presentaron AR muy alta y alta ( $74 \%$ y $16 \%$, respectivamente). Sólo un mínimo porcentaje de estos adolescentes presentaron niveles bajos de ansiedad (Tabla 6).

Tabla 7.

Ansiedad de adolescentes 16 a 18 años, Sex = Femenino; 154 sujetos

\begin{tabular}{ccccc}
\hline ADOLESCENTES & \multicolumn{2}{c}{ ANSIEDAD ESTADO } & \multicolumn{2}{c}{ ANSIEDAD RASGO } \\
\hline CATEGORÍAS & FREC & $\%$ & FREC & $\%$ \\
\hline Muy alta & 42 & 27 & 94 & 61 \\
Alta & 60 & 39 & 40 & 26 \\
Media & 42 & 27 & 16 & 10 \\
Baja & 10 & 6 & 4 & 3 \\
Total & 154 & 100 & 154 & 100 \\
\hline
\end{tabular}

En el caso de las mujeres adolescentes con el mismo rango de edad, también se encontraron altos niveles de ansiedad, principalmente de AR con un $\mathbf{8 7} \%$ en las categorías muy alto y alto (61 \% y $26 \%$, respectivamente) así como de AE con un $66 \%$ en las categorías muy altos y alto ( $27 \%$ y $39 \%$, respectivamente). De manera similar, un mínimo porcentaje de ellas presentaron bajos niveles de ansiedad, aunque ligeramente más alta que los varones (Tabla 7)
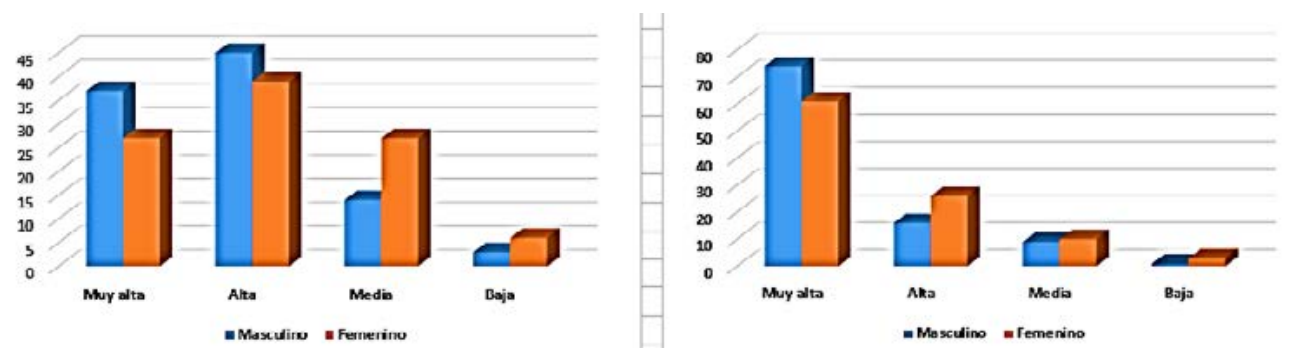

Figura 1. Resultados de ansiedad en adolescentes (de 16 a 18 años) 
En el comparativo, se puede observar un nivel más alto de $\mathbf{A E}$ y $\mathbf{A R}$ en el sexo masculino, ubicándose en las categorías muy alta. En la categoría alta se observa una AE proporcional para los dos sexos, pero ligeramente superior en el sexo masculino. En AR la categoría alta se encuentra ligeramente más alta en el sexo femenino (Figura 1). Se debe tener en cuenta que, para el rango de edad de 16 a 18 años, hay mayor proporción de sexo femenino que del sexo masculino (154 vs 91 ).

Tabla 8.

Ansiedad Adultos de 19 años o más, Sex = Masculino

\begin{tabular}{ccccc}
\hline ADULTOS & \multicolumn{2}{c}{ ANSIEDAD ESTADO } & \multicolumn{2}{c}{ ANSIEDAD RASGO } \\
\hline CATEGORÍAS & FREC & $\%$ & FREC & $\%$ \\
\hline Muy alta & 32 & 52 & 46 & 74 \\
Alta & 22 & 35 & 11 & 18 \\
Media & 7 & 11 & 5 & 8 \\
Baja & 1 & 2 & 0 & 0 \\
Total & 62 & 100 & 62 & 100 \\
\hline
\end{tabular}

Para el rango de edad de $\mathbf{1 9}$ años o más (adultos, baremos STAI) del sexo masculino, también se encontraron niveles altos de ansiedad. De acuerdo con la Tabla 8 , el $87 \%$ de los sujetos presentaron niveles muy altos y altos (52\% y $35 \%$, respectivamente) de Ansiedad Estado; y, un 92 \% presentaron niveles muy altos y altos (74\% y $18 \%$, respectivamente) de Ansiedad Rasgo. Un mínimo porcentaje presentaron niveles bajos de ansiedad.

Tabla 9.

Ansiedad Adultos de 19 años o más, Sex = Femenino

\begin{tabular}{ccccc}
\hline ADULTOS & \multicolumn{2}{c}{ ANSIEDAD ESTADO } & \multicolumn{2}{c}{ ANSIEDAD RASGO } \\
\hline CATEGORÍAS & FREC & $\%$ & FREC & $\%$ \\
\hline Muy alta & 19 & 30 & 38 & 59 \\
Alta & 33 & 52 & 13 & 20 \\
Media & 7 & 11 & 6 & 9 \\
Baja & 5 & 8 & 7 & 11 \\
Total & 64 & 100 & 64 & 100 \\
\hline
\end{tabular}

En el sexo femenino, el $82 \%$ presentó niveles muy altos y altos (30\% y $52 \%$, respectivamente) en $\mathbf{A E}$ y un $\mathbf{7 9} \%$ presentaron niveles muy altos y altos (59\% y $\mathbf{2 0} \%$, respectivamente) en AR. Porcentajes menores se encontraron en las categorías bajas, siendo más bajas en AE que en AR (Tabla 9). 


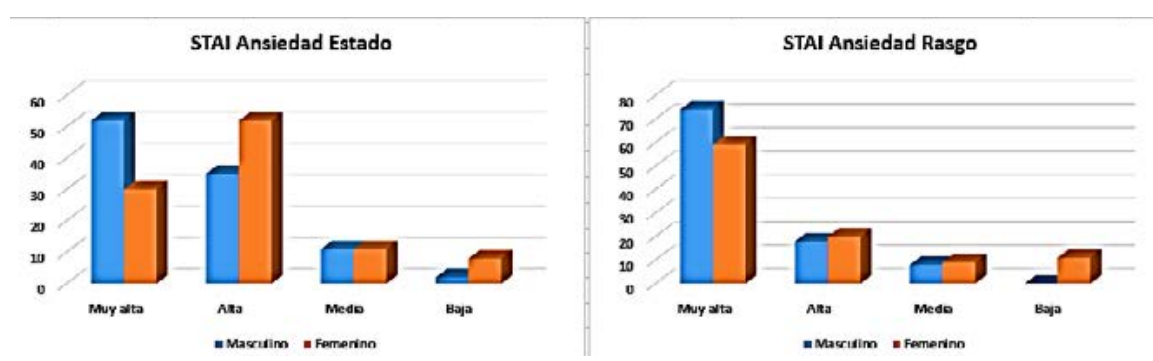

Figura 2. Resultados de ansiedad adultos (de 19 años o más)

En el comparativo se observa que, en la categoría muy alta tanto para ansiedad estado como para ansiedad rasgo, es más representativa en el sexo masculino, siendo superior en AE. Mientras que, en la categoría alta se puede observar una presencia mayor de ansiedad estado en el sexo femenino. En ansiedad rasgo la categoría alta es proporcional a los dos sexos, ligeramente superior en el sexo femenino, pero con pocos casos (Figura 2).

\subsection{Magnitud del tamaño del efecto de acuerdo con el sexo y edad}

Tabla 10.

Tamaño del efecto para muestras independientes del sexo sobre la ansiedad

\begin{tabular}{ccccc}
\hline \multicolumn{5}{c}{ ANSIEDAD } \\
\hline \multicolumn{3}{c}{ ESTADO } & \multicolumn{2}{c}{ RASGO } \\
\hline SEXO & MASCULINO & FEMENINO & MASCULINO & FEMENINO \\
\hline M & 29,28 & 28,01 & 30,45 & 32,53 \\
DE & 6,014 & 5,880 & 6,323 & 6,685 \\
t-student & 2,032 & & $-3,011$ & \\
p & 0,043 & & 0,003 & \\
\hline Cohen & & 0,214 & & 0,318 \\
\hline
\end{tabular}

Al analizar las medias encontramos que la diferencia entre sexo por ansiedad es mínima, siendo un poco más alta para el sexo masculino en ansiedad estado, estadísticamente significativo como indica el valor $p(0,43)$, e igual resultado se obtiene en cuanto a la ansiedad rasgo, siendo esto estadísticamente significativo como indica el valor $\mathrm{p}(0,03)$.

Para confirmar se calculó el tamaño del efecto para muestras independientes, encontrando que el tamaño del efecto fue pequeño para los casos de ansiedad estado $d=0,214(95 \%$ IC $0,41-2,5)$ y ansiedad rasgo $d=0,318$ $(95 \%$ IC $-3,43 ;-0,72)$, lo que indica que el impacto del confinamiento es bajo 
en los niveles de la ansiedad con relación al sexo, en especial para el sexo masculino (Tabla 10).

Tabla 11.

Tamaño del efecto para muestras independientes sobre la ansiedad

\begin{tabular}{|c|c|c|c|c|}
\hline \multirow[b]{3}{*}{ EDAD } & \multicolumn{4}{|c|}{ ANSIEDAD } \\
\hline & \multicolumn{2}{|c|}{ ESTADO } & \multicolumn{2}{|c|}{ RASGO } \\
\hline & DE 16 A 18 AÑOS & DE 19 AÑOS O MÁS & DE 16 A 18 AÑOS & DE 19 AÑOS O MÁS \\
\hline $\mathrm{M}$ & 28,49 & 28,62 & 31,64 & 31,72 \\
\hline $\mathrm{DE}$ & 6,037 & 5,831 & 6,323 & 7,161 \\
\hline t-student & $-0,198$ & & $-0,107$ & \\
\hline$p$ & 0,844 & & 0,915 & \\
\hline$\Delta$ Cohen & \multicolumn{2}{|c|}{0,022} & \multicolumn{2}{|c|}{0,012} \\
\hline
\end{tabular}

Al revisar las medias de los grupos de edad con relación a la ansiedad, encontramos que la diferencia es mínima, ligeramente más alta para el rango de 19 años o más, siendo esto estadísticamente significativo como indica el valor $p$ $(0,84)$ para Ansiedad Estado y estadísticamente significativo como indica el valor $p(0,915)$ para Ansiedad Rasgo.

Para confirmar lo descrito arriba, se calculó el tamaño del efecto para muestras independientes, encontrando que el tamaño del efecto fue pequeño $d=0,022$ (95 \% IC -1,416; 1,157) para el caso de ansiedad estado y para ansiedad rasgo $d=0,012$ (95 \% IC -1,504; 1,349), lo que indica el impacto del confinamiento es bajo en los niveles de la ansiedad estado con relación a la edad en especial para el rango de edad entre 19 años o más.

Encontramos entonces que, el impacto del confinamiento sobre los niveles de ansiedad (Estado-Rasgo) es bajo, y su elevación puede deberse a otros efectos asociados no considerados en el presente estudio.

\subsection{Estimación del riesgo de ansiedad, por grupos de edad y sexo.}

Tabla 12.

Estimación de riesgo $A E$, sexo masculino

\begin{tabular}{cccc}
\hline & \multicolumn{3}{c}{ INTERVALO DE CONFIANZA AL 95 \% } \\
\hline & VALOR & INFERIOR & SUPERIOR \\
\hline Razón de las ventajas para edad presente (De 16 a 18 años / De 19 & 1,109 & 0,467 & 2,636 \\
$\begin{array}{c}\text { años o más) } \\
\text { Para la cohorte Riesgo AE = Ausencia }\end{array}$ & 1,090 & 0,530 & 2,242 \\
Para la cohorte Riesgo AE = Presencia & 0,983 & 0,850 & 1,136 \\
N de casos válidos & 153 & & \\
\hline
\end{tabular}


Al aplicar el estadístico Odds Ratio (OR), retrospectivo, con un intervalo de confianza del 95 \% para realizar la estimación de riesgo por grupos de participantes, se halló que los varones adolescentes tienen 1,11 veces más riesgo de presentar $\mathrm{AE}$ que los adultos mayores de 19 años, sin embargo, al hallárseles un $\mathrm{OR}=0,983$ podría actuar como factor protector para ellos (Tabla 12).

Tabla 13.

Estimación de riesgo $\mathrm{AE}$, sexo femenino.

\begin{tabular}{cccc}
\hline & \multicolumn{2}{c}{ INTERVALO DE CONFIANZA AL 95 \% } \\
\hline $\begin{array}{c}\text { Razón de las ventajas para edad presente (De 16 a 18 años / De 19 } \\
\text { años o más) }\end{array}$ & VALOR & INFERIOR & SUPERIOR \\
\hline Para la cohorte Riesgo AE = Ausencia & $\mathbf{2 , 2 0 9}$ & $\mathbf{1 , 0 8 5}$ & 4,498 \\
Para la cohorte Riesgo AE = Presencia & 1,801 & 1,033 & 3,140 \\
N de casos válidos & $\mathbf{0 , 8 1 5}$ & 0,693 & 0,960
\end{tabular}

Un resultado relevante a tener en cuenta es el que se refiere al caso de las mujeres, pues al estudiar la fuerza de la asociación se obtuvo un OR de 2,2 con un intervalo de confianza de $95 \%$, por lo que se podría afirmar que tal asociación es significativa, por lo tanto, existe riesgo, donde las adolescentes presentan 2,2 veces más $A E$ que las adultas, representando un riesgo para su salud $(A E=$ presencia, 0,815$)$.

Este análisis confirma que la $\mathrm{AE}$ en el rango de edad comprendido de 16 a 18 años tiene mayor presencia en el sexo masculino que en el femenino (Figura 1).

Tabla 14.

\begin{tabular}{c|c|c|c}
\hline & \multicolumn{3}{c}{ INTERVALO DE CONFIANZA AL 95 \% } \\
\hline & VALOR & INFERIOR & SUPERIOR \\
\hline Razón de las ventajas para edad presente (De 16 a 18 años / & 1,251 & 0,398 & 3,929 \\
De 19 años o más) & 1,226 & 0,432 & 3,485 \\
Para la cohorte Riesgo AR = Ausencia & 0,980 & 0,887 & 1,084 \\
Para la cohorte Riesgo AR = Presencia & 153 & & \\
\hline N de casos válidos & &
\end{tabular}

De otra parte, para el caso de la AR, se encontró que los varones (de 16 a 18 años) presentan 1,3 veces aproximadamente más riesgo de presentar AR que los adultos (de 19 años o más). Al estudiar la fuerza de la asociación teniendo en cuenta que se obtuvo un OR de 1,3 con un intervalo de confianza de 0,5 se podría decir que la asociación NO es significativa, por lo tanto, NO existe ni representa riesgo para su salud. 


\section{Tabla 15.}

\begin{tabular}{cccc}
\hline & \multicolumn{3}{c}{ INTERVALO DE CONFIANZA AL 95 \% } \\
\hline & VALOR & INFERIOR & SUPERIOR \\
\hline Para la cohorte RiesgoAR = Presencia & 1,255 & 1,109 & 1,420 \\
N de casos válidos & 218 & & \\
\end{tabular}

Por su parte, en el caso del sexo femenino, al realizar análisis por columnas del riesgo de la presencia de la ansiedad como rasgo, se encontró que las adolescentes (de 16 a 18 años) presentan aproximadamente 1,3 veces más riesgo de presentar AR que las adultas (de 19 años o más); y, al estudiar la fuerza de la asociación teniendo en cuenta que se obtuvo un OR de 1,3 con un intervalo de confianza de 0,5 y observando que el valor obtenido se encuentra entre los rangos del intervalo, se podría decir que la asociación es significativa, por lo tanto, existe riesgo, las adolescentes presentan 2,2 veces más ansiedad estado que los que se encuentran en el rango de edad de 19 años o más, este representa un riesgo para su salud.

En este análisis, se confirma que la AR tiene mayor presencia en la categoría muy alto, en el sexo masculino que, en el femenino, en especial entre las edades de 16 a 18 años.

\section{DISCUSIÓN}

El objetivo del presente estudio buscó caracterizar la presencia de la ansiedad como trastorno del estado de ánimo, en estudiantes universitarios en situación de confinamiento obligatorio debido a la pandemia mundial del covid-19, pertenecientes a una Universidad estatal del Caribe colombiano.

Los resultados hallados muestran la presencia de altos niveles de ansiedad (estado y rasgo) en esta población, tanto en hombres como en mujeres, siendo ligeramente mayor en los hombres. Estos resultados son de preocupación si se considera que en promedio más del $80 \%$ de la población de estudiantes en aislamiento social obligatorio presentan altos niveles de ansiedad.

De igual manera, al estimar el riesgo de presentar ansiedad, se encontró que tanto hombres como mujeres presentaron un riesgo similar. Donde los varones adolescentes, presentan 1,3 veces aproximadamente más riesgo de presentar AR que los adultos, lo cual no representa riesgo para su salud. Mientras que, para el caso de las mujeres, las adolescentes presentan 2,2 veces más $A E$ que las adultas, lo cual, sí representa un riesgo para su salud. 
De hecho, la presencia de altos niveles de ansiedad en los estudiantes universitarios, durante el periodo de confinamiento obligatorio, estarían afectando la salud mental de la población universitaria y por ende esto podría estar afectando su aprendizaje académico, su autorregulación emocional y sus interacciones sociales.

Tal como se presentó en Colombia y el mundo en general desde el mes de marzo de 2020, el virus del covid-19 y sus secuelas de pandemia mortal y confinamiento obligatorio generaron en la población general un verdadero trauma psicológico expresado en incertidumbre, desconocimiento, miedos y desesperanzas, que la han llevado a sufrir diversos trastornos psicológicos como ansiedad, depresión, estrés postraumático y conductas autolesivas, entre otros. Al parecer, estos trastornos psicológicos afectan por igual a hombres y mujeres, pero con mayor presencia en los más jóvenes que en los adultos, lo que resalta el efecto negativo que viene generando esta situación en el desempeño de sus actividades cotidianas, en particular en sus actividades académicas de los estudiantes universitarios.

Estos resultados son coherentes con los hallados en otros estudios, como el de Ozamiz-Etxebarria, et al. (2020) en España, quienes reportan que la población más joven, al igual que aquellos con enfermedades crónicas, reportaron sintomatología de estrés, ansiedad y depresión más alta que el resto de la población, esperando que estas aumenten según vaya transcurriendo el tiempo de confinamiento. Los resultados del presente estudio confirman estas expectativas, dado que se hallaron elevados niveles de AE y AR a cinco meses después del inicio del confinamiento y distanciamiento social obligatorio en la población joven universitaria participante en el presente estudio.

También son coherentes con los informes de otros investigadores que alertaron sobre los efectos negativos en la salud mental de la medida de confinamiento obligatorio para afrontar la pandemia del covid-19, en especial en la población joven adolescente universitaria, la cual incluye a cerca del $30 \%$ de la población universitaria de la Institución Estatal participante, muy a pesar de haberse señalado a nivel mundial que esta población no estaría en riesgo de adquirir la enfermedad, poniéndose más cuidado en la población adulta mayor (Huarcaya, 2020; Quezada-Scholz, 2020; Ozamiz-Etxebarria, et al., 2020; Urzúa, et al., 2020; Wang et al., 2020; González-Jaimes, TejedaAlcántara, Espinosa-Méndez, Ontiveros-Hernández, 2020).

De manera análoga, son coherentes con los resultados hallados por SigüenzaCampoverde y Vílchez Tornero (2021) en Ecuador, los cuales aplicaron 
un cuestionario finalizando el ciclo académico (febrero 2020) y al final del siguiente ciclo académico (agosto 2020) volvieron aplicar el cuestionario de ansiedad, en el cual observaron que los niveles de ansiedad en los estudiantes habían aumentado en un 2,59 puntos con referencia a los primeros resultados antes del covid, es por esto que considera la aparición de la covid-19 como un factor influyente en el aumento de los niveles de ansiedad que los estudiantes manifiestan como malestar psicológico.

En el mismo sentido, es relevante el impacto de la ansiedad en la población de adolescentes universitarios, en especial en las mujeres adolescentes, quienes presentan 2,2 veces más riesgo de presentar $A E$ que las adultas, representando un riesgo para su salud mental (González-Jaimes, TejedaAlcántara, Espinosa-Méndez, Ontiveros-Hernández, 2020; Cobo-Rendón, Vega-Valenzuela y García Álvarez, 2020).

Uno de los factores explicativos podría deberse al exceso de información, información contradictoria y aislamiento social a que se ven sometidos los jóvenes, ávidos de información y contacto social, lo que generaría una percepción poco objetiva o distorsionada de las formas y estrategias de prevención contra el covid-19, tal como lo afirma Lazarus (1991), en el sentido que lo relevante no es el estresor mismo sino la percepción que la persona tiene sobre ello lo que causa el estrés o lo magnifica, distorsionando también sus expectativas y metas, que también pueden ser percibidas como desafíos o amenazas, incrementando sus niveles de ansiedad ante la pandemia

Finalmente, cabe destacar el hallazgo de que los hombres tienen mayor presencia de AE y AR que las mujeres, lo que implica poner una mayor atención en la población masculina en las actividades de prevención y promoción de la salud mental en las Universidades. Sin embargo, ambos tienen un riesgo similar de presentarlas, siendo este ligeramente mayor en las mujeres.

\section{CONCLUSIÓN}

En efecto, a manera de conclusión se puede afirmar que los jóvenes universitarios adolescentes presentan altos niveles de ansiedad, AE y $A R$, que pone en riesgo su salud mental y sus actividades académicas universitarias en periodo de confinamiento, ante lo cual se hace necesario diseñar y poner en práctica diversas estrategias de prevención y promoción de la salud mental desde la Institución universitaria, además de brindar información científica oportuna y suficiente que garantice la tranquilidad necesaria para el desempeño de sus 
actividades académicas cotidianas en el aislamiento social obligatorio en que permanecen, evitando la posibilidad de presentar conductas de evitación o escape que podrían incrementar la ansiedad, el miedo, la desesperación y la depresión (Quezada-Scholz, 2020; Talavera, 2020; Reimers y Schleicher, 2020).

Lo anterior, es urgente dado el contexto preocupante en el país, donde a la fecha de este informe se contabiliza un aproximado de 400 mil contagiados y más de 13.000 fallecidos, de todas las edades, por lo que la situación de confinamiento social obligatorio no cede en el país ni en las instituciones universitarias (MINSALUD, 2020).

Al respecto, un elemento importante de prevención dirigido a las Instituciones de Educación Superior ha sido señalado por la UNESCO-IELSALC (2020), por cuya relevancia y pertinencia se resume a continuación:

1. Difundir entre la comunidad universitaria única y exclusivamente informaciones y recomendaciones publicadas por las autoridades sanitarias nacionales y la Organización Mundial de la Salud, evitando el alarmismo o la propagación de rumores o noticias falsas.

2. Utilizar regularmente el sitio web y las redes sociales de la Universidad para informar oportuna y verazmente a la comunidad universitaria sobre el covid-19, las conductas a seguir preventivamente o en caso de contagio, los últimos avances en investigación, evitando actitudes y comportamientos racistas o discriminatorios.

3. Las Universidades que cuenten con facultades de medicina o escuelas de salud pública, facilitar el desarrollo de cursos de libre acceso sobre el covid-19, que contribuyan a una mayor calidad de la educación sanitaria de la población en general y a conformar una actitud positiva hacia la investigación científica.

Conflicto de interés: Los autores declaran que no tienen conflicto de intereses en el presente artículo.

Agradecimientos: Los autores agradecen la participación de todos los estudiantes de la Universidad del Magdalena que, estando confinados en sus casas, dedicaron una parte de su valioso tiempo para responder el Cuestionario enviado, haciendo posible la elaboración del presente artículo.

Financiamiento: El estudio fue financiado por los autores. 


\section{REFERENCIAS}

Barraza, A. y Jaick, A. (2011). Estrés, burnout y bienestar subjetivo. Investigaciones sobre la salud mental de los agentes educativos. CIIDIR-IPN, Unidad Durango - ReDIE - IUNAES. Primera edición. México. https://redie.mx/librosyrevistas/ libros/estres_burnout_y_bienestar_sujetivo.pdf

Cáceres, P. K. (2020). Educación virtual: Creando espacios afectivos, de convivencia y aprendizaje en tiempos de covid-19. CienciAmérica, 9(2). ISSN 1390-9592 ISSN-L 1390-681X. http://dx.doi.org/10.33210/ca.v9i2.284

Castillo Pimienta, C., Chacón de la Cruz, T. y Díaz-Véliz, G. (2016). Ansiedad y fuentes de estrés académico en estudiantes de carreras de la salud. Investigación en educación médica, 5(20), 230-237. https://doi.org/10.1016/j.riem.2016.03.001

Cobo-Rendón, Vega-Valenzuela y García Álvarez (2020). Consideraciones institucionales sobre la Salud Mental en universitarios frente al covid-19. http://dx.doi. org/10.33210/ca.v9i2.322

Ferrel, F., Ferrel, L., Cantillo, A., Jaramillo, J. y Jiménez, S. (2017). Variables académicas y sociodemográficas relacionadas con el Síndrome de Burnout, en estudiantes de Ingenierías y Ciencias de la Salud de una Universidad Estatal de Colombia. Psicogente, 20(38), 336-352. http://doi.org/10.17081/psico.20.38.2555

González-Jaimes, Tejeda-Alcántara, Espinosa-Méndez, Ontiveros-Hernández (2020). Impacto psicológico en estudiantes universitarios mexicanos por confinamiento durante la pandemia por covid-19. https://doi.org/10.1590/SciELOPreprints.756

Huarcaya, J. (2020). Consideraciones sobre la salud mental en la pandemia de covid-19. Rev Perú Med Exp., Salud Pública, 37(2): 327-34. https://doi. org/10.17843/rpmesp.2020.372.5419

Lazarus, R. S. (1991). Emotion and adaptation. Oxford University Press.

Ministerio de Salud. Situación actual: Nuevo Coronavirus (covid-19), 10 de agosto de 2020. https://www.minsalud.gov.co/salud/publica/PET/Paginas/covid-19_copia. aspx

Ortiz, J., Morocho, M., Tenezaca, Á., Torres, M., y Ugalde, D. (2014). Diagnóstico de la relación entre el uso excesivo de las TICs y síntomas depresivos y ansiedad en estudiantes de medicina, Universidad de Cuenca, Ecuador. Maskana, 5(2), 41-48. https://doi.org/10.18537/mskn.05.02.04

Ozamiz-Etxebarria, N., Dosil-Santamaría, M., Picaza-Gorrochategui, M., y Idoiaga-Mondragon, N. (2020). Niveles de estrés, ansiedad y depresión en la primera fase del brote del covid-19 en una muestra recogida en el norte de España. Cadernos de Saúde Pública, 36(4), e00054020. Epub April 30. https://doi. org/10.1590/0102-311×00054020

Quezada-Scholz, V. (2020). Miedo y psicopatología: la amenaza que oculta el covid-19. Facultad de Ciencias sociales, Universidad del Chile. http://www.facso.uchile.cl/ noticias/163245/miedo-y-psicopatologia-la-amenaza-que-oculta-el-covid-19

Reimers y Schleicher (2020). Un marco para guiar una respuesta educativa a la pandemia del 2020 de covid-19. Proyecto EDUCAR. https://globaled.gse.harvard. edu/files/geii/files/un_marco_para_guiar_una_respuesta_educativa_a_la_ pandemia_del_2020_del_covid-19_.pdf

Rivera, J. A., Cruz-Santos, A., y Rodríguez-Ríos, L. (2020). Demandas Tecnológicas, Académicas y Psicológicas en Estudiantes Universitarios durante la Pandemia por covid-19. Revista Caribeña de Psicología, 4(2), 176-185. https://doi.org/10.37226/ rcp.v4i2.4915

Sigüenza-Campoverde, W. y Vílchez-Tornero, J. (2021). Aumento de los niveles de ansiedad en estudiantes universitarios durante la época de pandemia de la 
covid-19. Revista Cubana de Medicina Militar, 50(1). http://scielo.sld.cu/pdf/mil/ v50n1/1561-3046-mil-50-01-e931.pdf

Spielberg, C.D., Gorsuch, R.L. y Lushene, R. E. (1997). STAI Cuestionario de Ansiedad Estado-Rasgo, $4^{\circ}$ edición revisada. Madrid. TEA. Publicaciones de Psicología Aplicada. https://dokumen.tips/documents/stai-ansiedad-estado-rasgo.html

Talavera. (2020). La educación en tiempos de pandemia: los desafíos de la escuela del siglo XXI. № 44, pp.176-187. http://www.grupocieg.org/archivos_revista/ Ed.44(176-187)\%20Hurtado\%20Tavalera_articulo_id650.pdf

UNESCO IESALC. (2020). El coronavirus covid-19 y la educación superior: impacto y recomendaciones. Recomendaciones de para las instituciones de educación superior (publicadas al inicio de la pandemia). https://www.iesalc.unesco. org/2020/04/02/el-coronavirus-covid-19-y-la-educacion-superior-impacto-y-recomendaciones/

Urzúa, A; Vera-Villarroel, P.; Caqueo-Urízar, A. y Polanco-Carrasco, R. (2020). La Psicología en la prevención y manejo del covid-19. Aportes desde la evidencia inicial. Terapia Psicológica 2020, 38(1), 103-118. https://teps.cl/index.php/teps/article/ view/273

Vanetza E. Quezada-Scholz. (2020). Miedo y psicopatología: la amenaza que oculta el covid-19. Departamento de Psicología, Facultad de Ciencias Sociales, Universidad de Chile. http://www.facso.uchile.cl/noticias/163245/miedo-y-psicopatologia-la-amenaza-que-oculta-el-covid-19

Wang, C., Pan, R., Wan, X., Tan, Y., Xu, L., Ho, C. y Ho, R. (2020). Immediate Psychological Responses and Associated Factors during the Initial Stage of the 2019 Coronavirus Disease (covid-19). Epidemic among the General Population in China. Res. Public Health (17) 17-29. https://www.mdpi.com/1660-4601/17/5/1729/htm

Wotjak, C. T., y Pape, H.-C. (2013). Neuronal circuits of fear memory and fear extinction. E-Neuroforum, 19(3), 47-56. https://doi.org/10.1007/s13295-0130046-0

Yin, R., Feng W., Wang T., Chen G., Wu T., Chen D., Lv, T., Xiang, D. (2020). Concomitant neurological symptoms observed in patient diagnosed with coronavirus disease 2019. J Med Virol. 92:1782-1784. https://onlinelibrary.wiley.com/doi/ epdf/10.1002/jmv.25888

Esta obra está bajo: Creative commons attribution 4.0 international license. El beneficiario de la licencia tiene el derecho de copiar, distribuir, exhibir y representar la obra y hacer obras derivadas siempre y cuando reconozca y cite la obra de la forma especificada por el autor o el licenciante.

\section{(cc) $\mathbf{B Y}$}

